\title{
UK Renal Registry 11th Annual Report (December 2008): Appendix F UK Renal Registry dataset specification
}

This appendix is available on the UK Renal Registry website only and the current version of this document can be found at www.renalreg.org. 